J. Phys. G: Nucl. Part. Phys. 18 (1992) 35-48. Printed in the UK

\title{
Higher baryon and winding numbers in the Nambu-Jona-Lasinio model
}

\author{
D Berg†, E Ruiz Arriolaf, F Grümmer† and K Goeke† \\ † Institut für Theoretische Physik II, Ruhr-Universität Bochum, D-4630 Bochum, \\ Federal Republic of Germany \\ $\ddagger$ Departamento de Fisica Moderna, Universidad de Granada, E-18071 Granada, \\ Spain
}

Received 17 June 1991

\begin{abstract}
A bosonized version of the SU(2) Nambu-Jona-Lasinio model is solved self-consistently for hedgehog fields on the chiral circle in the solitonic sector with baryon number $\bar{B}=2$. We consider explicitly the case in which the baryon number is raised by occupying $B$-bound valence quark states for different winding numbers $\omega$ of the chiral angle. It is found that only the cases $B \geqslant \omega$ allow the existence of self-consistent solutions. Fractional windings are also excluded in practice by the selfconsistency condition. For the description of nucleon properties the constituent mass has to be chosen around $400 \mathrm{MeV}$. There no solitons with higher winding numbers are found in contrast to the results of the Skyrme model. On the other hand, in this region the binding energy of the deuteron and the $\alpha$-particle can be roughly reproduced with winding number one.
\end{abstract}

\section{Introduction}

In modelling low-energy QCD, chiral effective Lagrangians have been proved, during the past few years, to describe many aspects of hadronic physics successfully. This is probably due to the fact that they incorporate the spontaneous breaking of chiral symmetry which is, presumably, the relevant mechanism of QCD at low energies. Among these theories the simplest one exclusively containing quark degrees of freedom is represented by the Nambu-Jona-Lasinio (NJL) model [1]. In fact, there have been some attempts claiming that a model of this kind can be motivated directly from QCD in some long wavelength approximation [2]. The model is non-renormalizable and thus a UV cut-off procedure has to be introduced $[3,4]$.

The partially bosonized version of this model supports self-consistent solitonic solutions [5], and hence has recently demanded much attention. In contrast to other soliton models the NJL model does not make a priori any assumption about the validity of the valence quark picture, i.e. whether the baryon charge has a topological or a non-topological origin. Actually, numerical calculations within the NJL model [6] show that, as far as the nucleon is concerned, the valence quarks dominate the sea quarks leading to a well defined valence quark picture. This feature is known to be fairly independent of the particular regularization scheme applied [7]. However these investigations have been limited up to now to the baryon number $B=1$ sector. The purpose of the present article is to investigate numerically and systematically the NJL 
model for higher winding and occupation numbers and to look for their relationship in a self-consistent manner.

The question of higher baryon numbers has already been addressed by different authors [ $[\overline{8}]$. Fistoricaily it was Skyrme who was the first to try to describe these states by increasing the winding number within a restricted variational ansatz [9], assuming that the baryon and winding numbers coincide. This connection was somehow clarified by the work of Goldstone and Wilczek [10], however it was in the adiabatic approximation. Various authors have recently questioned the validity of this approximation [11, 12]. Within the Skyrme model higher winding numbers have been considered both in the hedgehog case [13] and in a non-symmetric ansatz [14]. The result of these investigations are that, while there is no stable particle-like state with $\omega=2$ which is based on hedgehog symmetry, the non-symmetric ansatz shows a binding state with an approximately toroidal shape. In [15] the Gell-Mann-Levy sigma model has also been treated for different occupation numbers in the hedgehog case. As in the Skyrme model no stable state has been found.

As we have already mentioned the NJL model becomes well defined only after the introduction of a regularization function. In general it is known that, although the vacuum properties depend strongly on the particular regularization scheme, the soliton properties do not. In the present work the Schwinger proper time regularization method [16] will be used.

\section{Description of the model}

The $S U(2)$ NJL model written in terms of (up and down) quark spinor fields $\Psi$ containing scalar and pseudoscalar couplings reads

$$
\mathcal{L}_{\mathrm{NJL}}=\bar{\Psi} \mathrm{i} \not \partial \Psi+\frac{G}{2}\left[(\bar{\Psi} \Psi)^{2}+\left(\bar{\Psi} \mathrm{i} \gamma_{5} \tau \Psi\right)^{2}\right]-m_{0} \bar{\Psi} \Psi
$$

where the summation over $N_{\mathrm{c}}=3$ colour degrees of freedom is assumed implicitly. $G$ stands for the strength of the coupling and $m_{0}$ denotes the average up and down current quark mass. Except for the last term in (1) this Lagrangian is invariant under the $U(1)_{B} \otimes S U(2)_{V} \otimes S U(2)_{A}$ group. This leads to a set of Noether currents

$$
\begin{aligned}
& B_{\mu}=\bar{\Psi} \gamma_{\mu} \Psi \\
& V_{\mu}=\bar{\Psi} \gamma_{\mu} \tau / 2 \Psi \\
& A_{\mu}=\bar{\Psi} \gamma_{\mu} \gamma_{5} \tau / 2 \Psi
\end{aligned}
$$

which are, up to the last one, exactly conserved. In fact the baryon current $(2 a)$ and, more specifically, the corresponding charge will be the main subject of our work. For a more detailed exposition of both the model and its parameters we refer the reader to $[3-5]$.

Within the path integral approach the NJL action in Euclidean space can be equivalently transformed after bosonizaton and integrating out the quarks [4] into the 
following effective action

$$
\begin{aligned}
\mathcal{S}_{\mathrm{eff}} & =\mathcal{S}_{\mathrm{eff}}^{\mathrm{F}}+\mathcal{S}_{\mathrm{eff}}^{\mathrm{br}} \\
\mathcal{S}_{\mathrm{eff}}^{\mathrm{F}} & =-S p \ln \not D \\
\not D & =-\mathrm{i} \not \partial+g\left(\sigma+\mathrm{i} \gamma_{5} \tau \pi\right) \\
\mathcal{S}_{\mathrm{eff}}^{\mathrm{br}} & =-m_{\pi}^{2} f_{\pi} \int \mathrm{d}^{4} x \sigma
\end{aligned}
$$

where the quark-meson coupling constant $g$ is related to the constituent mass $M$ by $M=g f_{\pi}$. Following [4] we will consider classical meson fields only, where the meson fields are regarded as fulfilling the chiral circle condition

$$
\tilde{v}^{2}+\pi^{2}=f_{\pi}^{2}
$$

with $f_{\pi}=93 \mathrm{MeV}$ being the pion decay constant. Moreover, we regularize the UV divergencies in the real part in Euclidean space of the fermionic action by means of the Schwinger proper-time method. The imaginary part is known to vanish. This leads to

$$
S_{\text {eff }}^{\mathbf{F}}=\frac{1}{2} S p \int_{1 / \Lambda^{2}}^{\infty} \mathrm{d} \tau \frac{\mathrm{e}^{-\tau \not \not^{\dagger} \not \not p}}{\tau}
$$

In the static case the corresponding regularized onc-quark loop encrgy reads

$$
\begin{aligned}
E^{\mathrm{QL}} & =E_{\mathrm{F}}+E_{\mathrm{br}} \\
E_{\mathrm{F}} & =\frac{N_{\mathrm{c}}}{4 \sqrt{\pi}} \sum_{\lambda}\left[I_{3 / 2}\left(\epsilon_{\lambda}, \Lambda\right)-I_{3 / 2}\left(\epsilon_{\lambda}^{0}, \Lambda\right)\right] \\
E_{\mathrm{br}} & =-m_{\pi}^{2} f_{\pi} \int \mathrm{d}^{3} x\left(\sigma-f_{\pi}\right)
\end{aligned}
$$

where the eigenvalues $\epsilon_{\lambda}$ of the following single-particle Dirac Hamiltonian

$$
h=\frac{\boldsymbol{\alpha} \nabla}{\mathrm{i}}+g \beta\left(\sigma+\mathrm{i} \gamma_{5} \tau \pi\right)
$$

have been introduced, $\epsilon_{\lambda}^{0}$ corresponding to the vacuum case. The regularization function can be written in terms of the incomplete gamma function as follows

$$
I_{K}(M, \Lambda)=M^{2(K-1)} \Gamma\left(1-K,(M / \Lambda)^{2}\right)
$$

In our approach valence quark states appear by introducing a chemical potential $\mu$ as was done in [4]. This yields the following valence quark energy

$$
E_{\mathrm{val}}=N_{\mathrm{c}} \sum_{0<\epsilon_{\lambda}<\mu} \epsilon_{\lambda}
$$


corresponding to the following valence baryon number

$$
B(\mu)=\sum_{0<\epsilon_{\lambda}<\mu} 1 .
$$

This prescription corresponds to the occupation of the lowest positive energy eigenstates. In practical calculations the hedgehog ansatz on the chiral circle

$$
U(\boldsymbol{x})=\sigma(\boldsymbol{x})+\mathrm{i} \boldsymbol{\tau} \cdot \boldsymbol{\pi}(\boldsymbol{x})=f_{\pi} \mathrm{e}^{\mathrm{i} \boldsymbol{\tau} \cdot \hat{\boldsymbol{r} \theta}(\boldsymbol{r})}
$$

will be used. The winding number is then defined as

$$
\omega=\frac{1}{\pi}[\theta(r=0)-\theta(r=\infty)]
$$

As was shown in $[2,4,12]$ the corresponding Dirac single-particle Hamiltonian commutes both with the grand spin $\boldsymbol{K}=\boldsymbol{J}+\boldsymbol{T}$ and the parity operator II. Hence grand spin $K$, its projection $K_{3}$ and the parity $\pi$ are good quantum numbers. We will denote the Dirac eigenstates by $K^{\pi}$, each state having a degeneracy $2 K+1$. Following the method of Ripka and Kahana [12], the eigenvalues $\epsilon_{\pi_{K}}$ and their eigenfunctions are computed by diagonalizing the Dirac equation in a sufficiently large box.

\section{Properties of the $B=2$ system in a fixed profile}

In this section we investigate the behaviour of the NJL model for higher winding and occupation numbers. It is instructive to proceed first with given external profiles as input, leaving the self-consistent treatment for the subsequent section. We will consider for illustration the following linear profile

$$
\theta(r)= \begin{cases}\omega \pi(1-r / R) & 0 \leqslant r<R \\ 0 & R \leqslant r \leqslant \infty\end{cases}
$$

where $R$ denotes the soliton size and $\omega$ stands for the winding number. The general behaviour does not depend much on the particular profile function $[17,18]$.

\subsection{Dirac spectrum}

The diagonalization of the Dirac equation for the linear profile allows the determination of the corresponding spectrum as a function of the soliton size $R$. In figure 1 we depict the scaled energy eigenvalues $\epsilon_{\lambda}^{\prime}=\epsilon_{\lambda} / M$ plotted against the scaled soliton size $R^{\prime}=R M$ for different winding numbers. We have considered four different cases: $\omega=1,1.8,2$ and 3 . In figure $1(a)$ the usual $\omega=1$ case has been presented. As we see only the $0^{+}$state becomes negative for sufficiently large soliton size and afterwards approaches the lower continuum. Figure $1(b)$ shows an example for non-integer winding $\omega=1.8$. In this case there exists an additional bound state with opposite parity which crosses zero and which eventually might cross back again for infinitely large soliton sizes. We have not found any hint supporting this expectation, probably due to the numerical limitations related to those very large solitons. In figure $1(c)$ the case $\omega=2$ has been considered. In contrast to the former case both $0^{+}$and $0^{-}$cross zero and approach the lower continuum. Finally in figure $1(d)$ the bound spectrum for 
$\omega=3$ has been plotted. Three levels with grand spin zero and alternating parity cross and later on get closer to the lower continuum. In fact the observed trends seem to be in agreement with analytical calculations for very large solitons and integer winding number in the $K=0$ sector [2]. These considerations lead to the following formula for the spectral flow from the upper to the lower continuum for the sector $K^{\pi}=0^{+} ; 0^{-}$

$$
Z^{0^{ \pm}}=\frac{1}{2} \omega \pm \frac{1}{4}\left[1-(-1)^{\omega}\right]
$$

which allows the winding number to be identified with the baryon number, however only in the case of infinitely large solitons and integer winding numbers. As we will see below this will not always be the case for calculations with constituent masses $M \simeq 400 \mathrm{MeV}$, which are relevant for the description of the nucleon structure. It is noteworthy that the formula (13) also exhibits the alternating parity feature obtained in our numerical calculation.

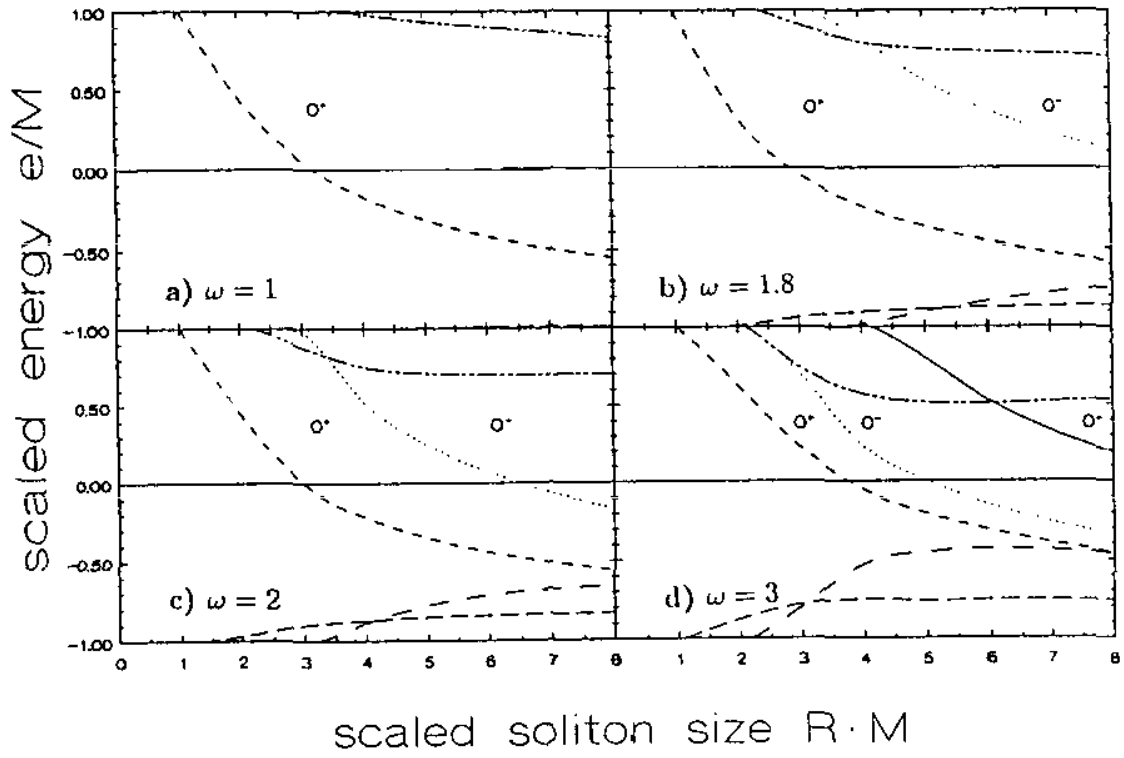

Figure 1. Spectrum of quark eigenstates for different values of the winding number: (a) $\omega=1 ;(b) \omega=1.8 ;(c) \omega=2$; and $(d) \omega=3$.

\subsection{Baryon density}

As we have already mentioned in the introduction the adiabatic approximation has been widely used in order to justify the identification of baryon number and topological charge. In this context the adiabatic approximation consists in using the first nonvanishing order of the gradient or heat kernel expansion of the baryon number density. A way of checking the validity of this approximation is by comparing the resulting baryon density to the exact one computed from the Dirac eigenfunctions. Indeed, the sea contribution to the exact baryon density can be computed to give

$$
\left\langle B_{0}(r)\right\rangle_{\mathrm{sea}}=-\frac{1}{2} \sum_{\lambda} \operatorname{sign}\left(\epsilon_{\lambda}\right) \tilde{\Psi}_{\lambda} \gamma_{0} \Psi_{\lambda}
$$


which corresponds to the spectral flow density [8]. The valence contribution to the baryon density arises from the bound orbits with positive quark eigenvalues $\epsilon_{\lambda}$ and can be computed via a chemical potential leading to

$$
\left\langle B_{0}(r)\right\rangle_{\text {val }}=\sum_{0<\epsilon_{\lambda}<\mu} \bar{\Psi}_{\lambda} \gamma_{0} \Psi_{\lambda}
$$

The adiabatic expansion of the sea contribution in the leading non-vanishing order can be written in terms of the chiral angle $\theta(r)$ in a simple way:

$$
\left\langle B_{0}(r)\right\rangle_{\mathrm{ad}}=-\frac{1}{2 \pi^{2}} \frac{\sin ^{2} \theta}{r^{2}} \frac{\mathrm{d} \theta}{\mathrm{d} r}
$$

In the case $\omega=1$ this comparison has been done in [12] showing a good agreement between the exact and approximate calculations. In figure 2, we present a similar comparison for $\omega=2$ for two values of the constituent mass and for the particular scaled soliton size $R^{\prime}=4$. Figure $2(a)$ corresponds to a situation in which for the exact calculation both valence levels are above zero and hence are occupied explicitly. Figure 2(b) on the contrary reflects the case where these two levels are well below zero. In these two cases we confirm the results found by Ripka et al, namely that the adiabatic approximation seems to work well for the baryon density in a wide range of constituent masses. We have checked that this conclusion does not depend strongly on the particular value of the scaled soliton size.

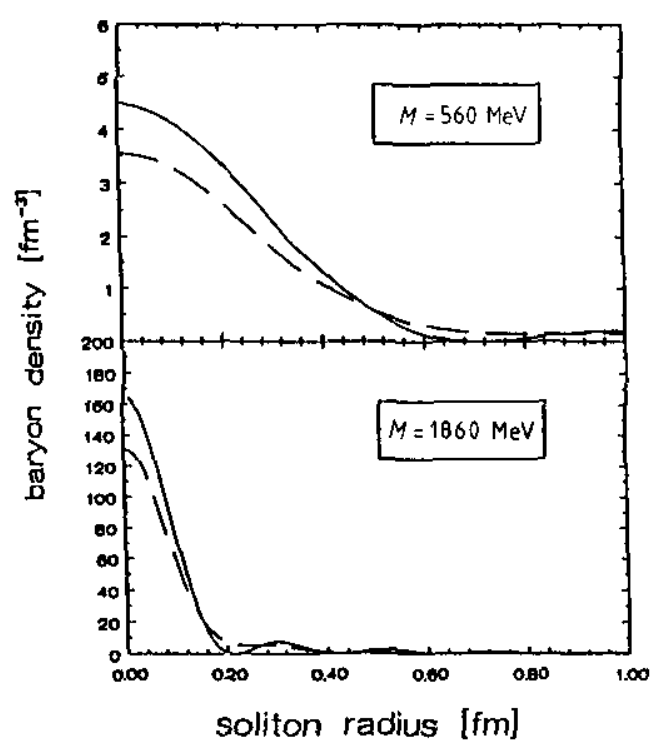

Figure 2. Exact (broken curve) and adiabatic (full curve) results for the baryon density with $B=\omega=2$ for $M=560 \mathrm{MeV}$ and $M=1860 \mathrm{MeV}$ calculated in the linear profile with scaled soliton size $R^{\prime}=4$. 


\subsection{Energy profiles}

Using a fixed profile, the total energy may be evaluated in terms of the soliton size $R$ and the winding number $\omega$ for a fixed value of the constituent mass and of the baryon number. This is done in the first part of this paragraph for the profile of (12). Next, a derivative expansion is applied with the quark-loop energy and compared to the exact calculation.

\subsection{Exact calculation}

Once the Dirac eigenstates have been determined one can use (5) and (8) in order to evaluate the total energy for the linear profile. It is instructive to do this by varying not only the soliton size but also the winding number $\omega$ for non-integer values. It is important to realize that in principle in the present non-topological model there is no particular reason to choose integer numbers only. However, as we will see later only this choice leads to local minima of the energy. In figure 3 we present the total energy (valence plus sea) surface for different values of the constituent mass and the baryon number as a function of the soliton size and the winding number. Apparently the present model shows finite energies even in the case of non-integer winding numbers. However, local minima in the energy appear only for integer winding numbers. For sufficiently large constituent masses the number of minima coincides with the baryon number for which the calculation is performed. For instance, in figure $3(a)$ the usual case $B=1$ has been considered. For a sufficiently large constituent mass a unique minimum at $\omega=1$ takes place. A transverse cut along the $R$-direction at $\omega=1$ corresponds to the results given in [4]. In the $B=2$ sector and for $M>560 \mathrm{MeV}$ one obtains two minima: one for $\omega=1$ and a second one at $\omega=2$. For increasing constituent mass the steepness of the second minimum grows. This can be seen in the sequence depicted in figures $3(b), 3(c)$ and $3(d)$. Of special interest is the transition from figure $3(c)$ to figure $3(d)$. There the minimal energy at double winding becomes lower than the energy with single winding, for those constituent masses higher than $M \simeq 900 \mathrm{MeV}$ the first valence orbital of the Dirac spectrum has already crossed the zero line. As we will see later, this behaviour will also be reflected in the self-consistent case.

\subsection{Comparison with the derivative expansion}

In the case of slowly varying meson fields or big soliton sizes the total energy can be represented for integer winding numbers by a power series expansion [18] as follows

$$
E_{\mathrm{ad}}=a R+\sum_{n=1}^{\infty} \frac{b_{n}}{R^{2 n+1}}
$$

For proper time regularization, this is done in [20]. The first two coefficients appearing in equation (17) can be determined uniquely in terms of the chiral angle $\theta$ and of the proper time cut-off $\Lambda$ yielding for $a$

$$
a R=2 \pi M^{2} \int_{0}^{\infty} \mathrm{d} r\left[r^{2} \theta^{2}+2 \sin ^{2} \theta\right]
$$



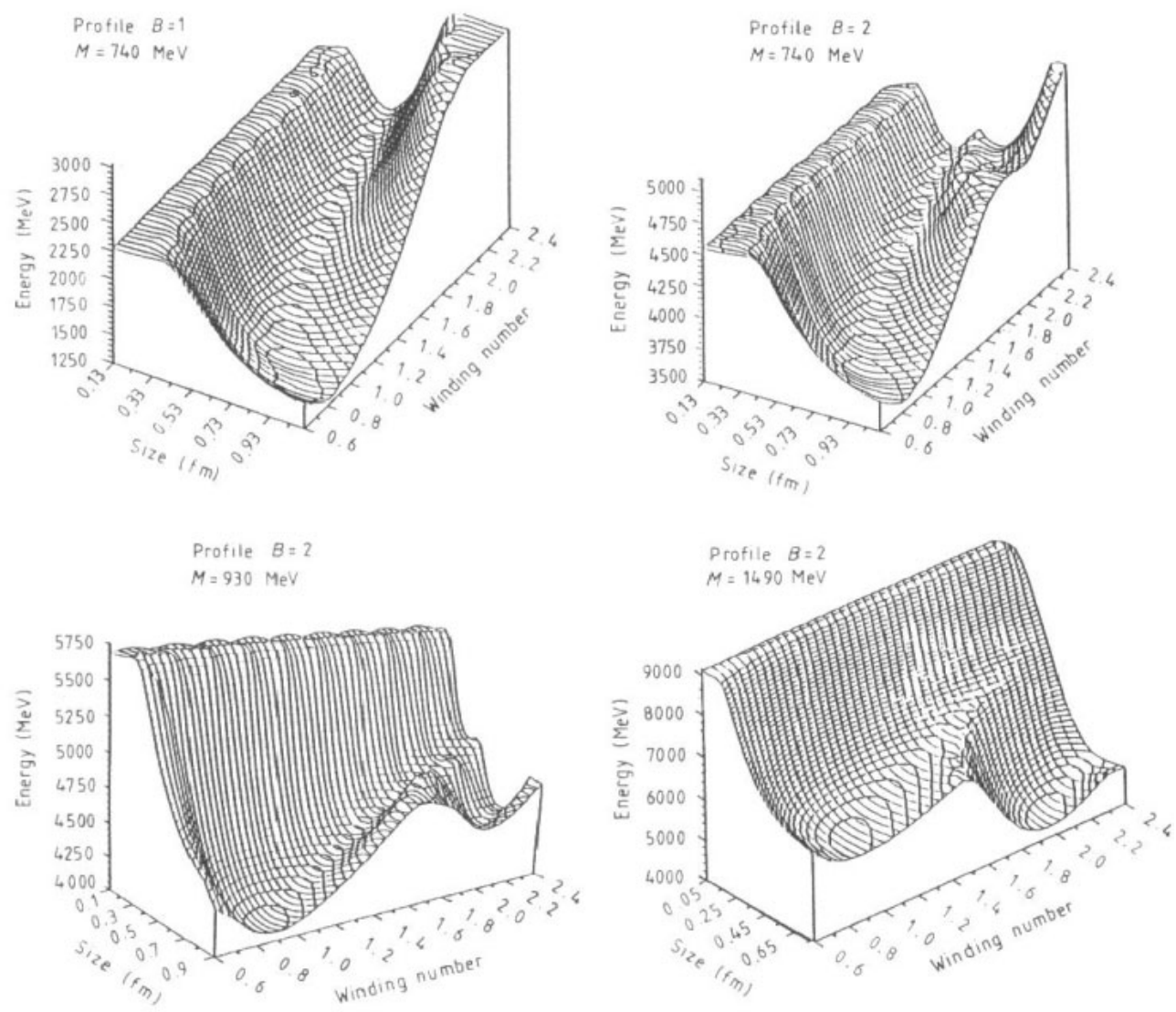

Figure 3. (Sea plus valence) energy surface calculated in the linear profile for different values of the baryon number and the constituent mass.

For $b_{1}$ one obtains the following two contributions denoted by $b^{\prime}, b^{\prime \prime}$ :

$$
\begin{aligned}
& b_{1}=b^{\prime}+b^{\prime \prime} \\
& \frac{b^{\prime}}{R}=\frac{M^{4}}{\pi} \int_{0}^{\infty} \mathrm{d} r\left\{\Gamma\left(2, \frac{M^{2}}{\Lambda^{2}}\right) \frac{\theta^{\prime 2}}{8}\left[\theta^{\prime 2} r^{2}+12 \sin ^{2} \theta\right]-\Gamma\left(1, \frac{M^{2}}{\Lambda^{2}}\right)\right. \\
& \left.\times\left[\frac{r^{2}}{4}\left(\theta^{\prime 4}+\theta^{\prime 2}\right)+\theta^{\prime 2}\left(1+\sin ^{2} \theta\right)+\theta^{\prime \prime}\left(\theta^{\prime} r-\frac{1}{2} \sin (2 \theta)\right)\right]\right\} \\
& \frac{b^{\prime \prime}}{R}=\frac{M^{4}}{\pi} \int_{0}^{\infty} \mathrm{d} r\left\{\left[\Gamma\left(2, \frac{M^{2}}{\Lambda^{2}}\right)-\Gamma\left(1, \frac{M^{2}}{\Lambda^{2}}\right)\right] \frac{\sin ^{4} \theta}{r^{2}}+\Gamma\left(1, \frac{M^{2}}{\Lambda^{2}}\right)\right. \\
& \left.\times\left[\frac{\theta^{\prime} \sin (2 \theta)}{r}-\frac{\sin ^{2}(2 \theta)}{4 r^{2}}\right]\right\} \text {. }
\end{aligned}
$$

We have checked that in the case of an integer winding number the exact energy can indeed be approximated by these expressions. We will also consider the possibility of non-integer winding numbers. A direct calculation shows, however, that the coefficient $b^{\prime \prime}$ diverges in this case. This resembles the situation of the topological Skyrme model. 
As is well known the requirement of finite soliton energy configurations enforces the winding number to be integer in that approximation.

The fact, that for non-integer winding numbers the coefficient $b^{\prime \prime}$ diverges, whereas the exactly evaluated energy is finite, is not a contradiction. Apparently, the gradient expansion is not a convergent expansion for non-integer winding numbers. One can see this explicitly in the following way: The coefficients $b_{n}$ of (17) are related to the gradients of the mesonic fields by

$$
\cdot b_{n} \sim \int \mathrm{d}^{3} x(\not \partial U)^{n+1}
$$

where the expression $U(x)$ in the hedgehog ansatz is given by (10). A term of $n$th order contains therefore

$$
\int \mathrm{d} r r^{2}\left[\frac{\partial}{\partial x_{i}}(\hat{r} \pi(r))\right]^{n+1}=\int \mathrm{d} r r^{2}\left[\frac{\pi(r)}{r}\right]^{n+1}
$$

which diverges for $n>1$ if $\pi(r)$ does not vanish at $r=0$. Therefore a gradient expansion only makes sense for integer winding numbers.

\section{Self-consistent solutions}

The minimization of the NJL action for static hedgehog configurations leads to the following equation for the chiral angle, if the non-linear model is considered $[5,6]$

$\tan \theta(r)=\frac{N_{c} g\left[\sum_{0<\epsilon_{\lambda}<\mu} \hat{r} \rho_{\lambda}(r)-\frac{2}{4 \sqrt{\pi}} \sum_{\lambda} \hat{r} \rho_{\lambda}(r) \epsilon_{\lambda} I_{1 / 2}\left(\epsilon_{\lambda}, \Lambda\right)\right]}{N_{c} g\left[\sum_{0<\epsilon_{\lambda}<\mu} \rho_{\lambda}^{0}(r)-\frac{2}{4 \sqrt{\pi}} \sum_{\lambda} \rho_{\lambda}^{0}(r) \epsilon_{\lambda} I_{1 / 2}\left(\epsilon_{\lambda}, \Lambda\right)\right]-4 \pi m_{\pi}^{2} f_{\pi}}$

$\rho_{\lambda}^{0}(r)=\int \mathrm{d} \Omega \bar{\Psi}_{\lambda}(r, \Omega) \Psi_{\lambda}(r, \Omega)$

$\rho_{\lambda}(r)=\int \mathrm{d} \Omega \bar{\Psi}_{\lambda}(r, \Omega)\left(\mathrm{i} \gamma_{5} \tau\right) \Psi_{\lambda}(r, \Omega)$

Following $[5,6]$ this equation can be solved iteratively until self-consistency is reached for a given baryon number. As input we consider for the first iteration the linear profile considered in (12) which besides the soliton size contains the winding number as parameters. We have tried different input integer and non-integer winding numbers for fixed baryon numbers. No self-consistent solutions with fractional winding have been found. This confirms the results obtained in section 3 for fixed soliton profiles. Whatever input $\omega$ is used the self-consistency condition (22) always drives the solution to a final integer $\omega$. For integer winding number we have considered the cases $(B=$ $1, \omega=1),(B=2, \omega=1),(B=1, \omega=2)$ and $(B=2, \omega=2)$. Solitonic solutions have only been obtained in the first, second and fourth cases. In fact the first case has been largely investigated in $[5,6]$ and we regard it here only for the sake of comparison. The third case did not exhibit any self-consistency minimum. This can be understood 
Table 1. Energy (MeV) and RMs baryon radius (fm) (in brackets) for different baryon and winding numbers. (Here occupied states are denoted by [].)

\begin{tabular}{lllll}
\hline $\mathrm{g}$ & $M(\mathrm{MeV})$ & $\underline{B-1}, \ldots=1$ & $B=2, \omega=1$ & $D=2, i=2$ \\
\hline 4 & 372 & $1260(0.77)\left[0^{+}\right]$ & $2390(1.48)\left[0^{+}, 0^{-}\right]$ & - \\
5 & 465 & $1242(0.63)\left[0^{+}\right]$ & $2655(1.17)\left[0^{+}, 0^{-}\right]$ & - \\
6 & 558 & $1190(0.57)\left[0^{+}\right]$ & $2903(1.73)\left[0^{+}, 1^{+}\right]$ & $3780(0.88)\left[0^{+}, 0^{-}\right]$ \\
7 & 651 & $1186(0.53)\left[0^{+}\right]$ & $3135(1.39)\left[0^{+}, 1^{+}\right]$ & $3776(0.84)\left[0^{+}, 0^{-}\right]$ \\
8 & 744 & $1155(0.50)\left[0^{+}\right]$ & $3360(1.17)\left[0^{+}, 1^{+}\right]$ & $3748(0.82)\left[0^{+}, 0^{-}\right]$ \\
9 & 837 & $1124(0.47)\left[0^{+}\right]$ & $3580(1.02)\left[0^{+}, 1^{+}\right]$ & $3708(0.81)\left[0^{+}, 0^{-}\right]$ \\
10 & 930 & $1094(0.45)\left[0^{+}\right]$ & $3800(0.92)\left[0^{+}, 1^{+}\right]$ & $3658(0.79)\left[0^{+}, 0^{-}\right]$ \\
\hline
\end{tabular}

easily in the light of figure 3 : for $B=1$ only one minimum appears, namely at $\omega=1$. We must note that no solitons have been found in the case $\omega>B$.

In table 1, we present our results for the self-consistent energies and their corresponding RMS baryon radii as a function of the constituent quark mass. Our results for $B=1$ coincide with those of [6]. As in that work both the energy and the RMS radius decrease monotonically with the constituent mass. The case $B=2, \omega=1$, on the contrary, shows an increasing soliton energy and a decreasing radius. In fact this case corresponds qualitatively to the situation sketched in figure 1(a) for a fixed profile: one valence orbital $0^{+}$becomes negative whereas the second one stays close to the mass threshold. As a result the total energy is shifted by approximately $N_{\mathrm{c}} M$ with respect to the $B=1, \omega=1$ energy. It should be mentioned that in all cases only the lowest energy orbitals were occupied, as it is drawn from the use of the chemical potential. One should notice that the lowest encrgy eigenvalue does not always have the same quantum number. This can be seen in table 2, where the total energy of the system is presented for self-consistent solutions, if two fixed states are occupied. For instance, for $M<560 \mathrm{MeV}$ the lowest $B=2, \omega=1$ configuration is obtained by occupying the $0^{+}$and $0^{-}$orbitals. From $M=560 \mathrm{MeV}$ onwards the lowest energy state is given by the occupation of the $0^{+}$and $1^{+}$orbitals. We have also considered the case in which the $0^{+}$and $1^{-}$orbitals are occupied. We have found that this configuration never has the lowest energy. Finally the $B=2, \omega=2$ solution shows a similar behaviour to that of $B=1, \omega=1$, namely a decreasing soliton size and energy. For illustration in figure 4 we have plotted both the resulting self-consistent meson fields and the related baryon density in the last case for the particular value of the constituent mass $M=560 \mathrm{MeV}$, showing the extended structure of such a system. In figure $4(a)$ one can see the typical two knots characterizing the double winding number. Figure $4(b)$ shows the baryon density together with the different contributions to it, namely the sea and the two valence quark contributions.

In figure 5 we present the dependence of the general behaviour of the different configurations with $B=2$ on the constituent mass $M=g f_{\pi}$. For comparison we also plot the energy of six free quarks and twice the energy for the $B=1$ solution. From an energy point of view it becomes clear that for $M<420 \mathrm{MeV}$ all configurations may decay into six free quarks. For higher constituent masses the system with single winding and $B=2$ can decay into two separated systems with $B=1$. From $M=$ $560 \mathrm{MeV}$ onwards $B=2$ solutions with double winding exist and may also decay for $M<630 \mathrm{MeV}$ in any of the previously mentioned systems. However, for large constituent mass $M \simeq 880 \mathrm{MeV}$ this system only remains unstable against decay into two separated $B=1$ solitons. 
Table 2. Energy $(\mathrm{MeV})$ for different occupied upper orbitals in the case $(B=2$, $\omega=1$ ).

\begin{tabular}{lllll}
\hline $\mathrm{g}$ & $M(\mathrm{MeV})$ & {$\left[0^{+}, 0^{-}\right]$} & {$\left[0^{+}, 1^{-}\right]$} & {$\left[0^{+}, 1^{+}\right]$} \\
\hline 4 & 372 & 2390 & 2403 & 2400 \\
5 & 465 & 2655 & 2669 & 2659 \\
6 & 558 & 2912 & 2926 & 2903 \\
7 & 651 & 3165 & 3177 & 3135 \\
8 & 744 & 3417 & 3423 & 3360 \\
9 & 837 & 3670 & 3663 & 3580 \\
10 & 930 & 3924 & 3892 & 3800 \\
\hline
\end{tabular}
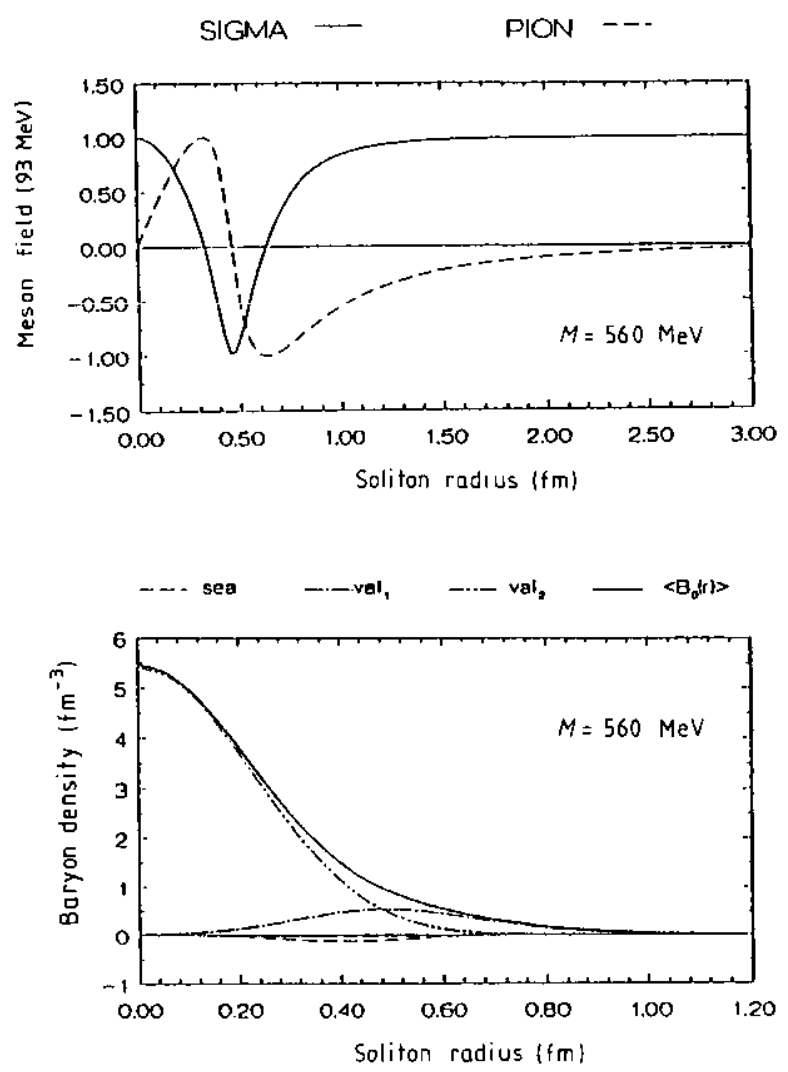

Figure 4. Self-consistent mesonic fields (a) and the corresponding baryon density (b) for the $B=2$ system with double winding. The latter is decomposed in its valence and sea contributions.

\section{Potential energies}

If we consider, as in the Skyrme model [21], the $B=2$ system as two baryon hedgehogs at zero separation $d$, we obtain the potential energy of two baryons at $d=0$

$$
V(d=0)=E_{B=n}-n E_{B=1}
$$

if $n=2$. This can be evaluated both for the single and double winding cases. For the single winding case, the potential energy of a system with four baryons has also been 


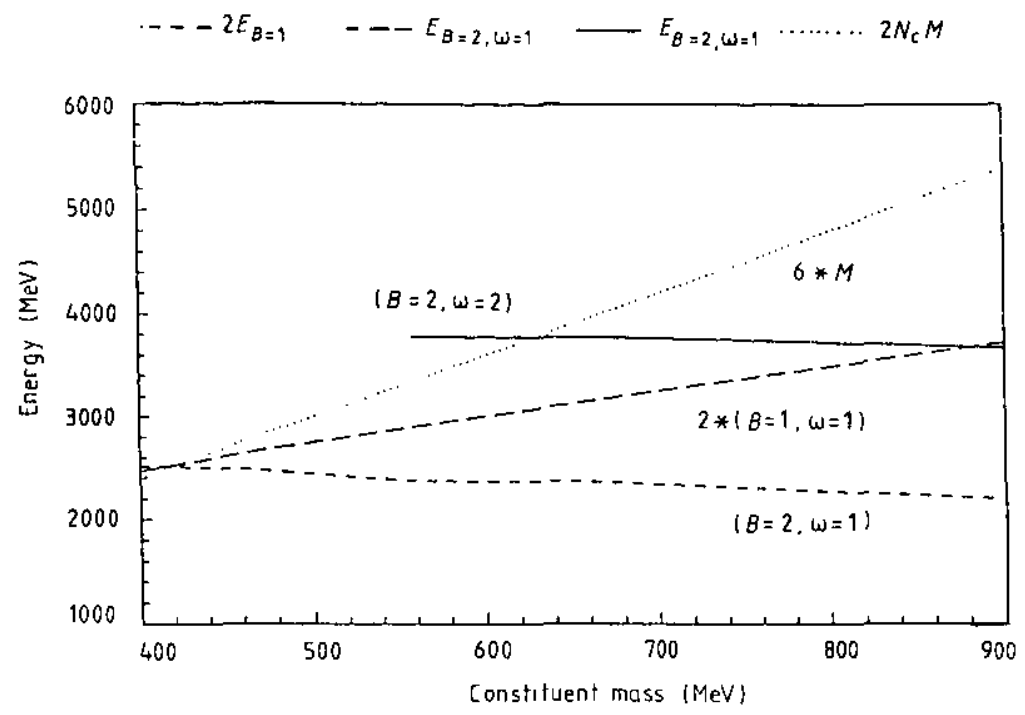

Figure 5. Total energy of different self-consistent solutions compared to the energy of six free quarks (dotted line).

calculated. We present in table 3 the dependence obtained for the potential energy as a function of the constituent mass. As we see the single winding case depends rather strongly on the particular value of $M$, whereas the double winding case remains nearly constant. In fact, for small constituent masses the single winding case leads to a negative potential energy for $B=2$; the same happens for the $B=4$ system. If one tries to reproduce the experimental deuteron binding energy of $2 \mathrm{MeV}$, a linear extrapolation leads to a constituent mass of $M_{2}=411 \mathrm{MeV}$. The $B=4$ system, taken as an approximation for the $\alpha$-particle, leads to a constituent mass of $M_{4}=400 \mathrm{MeV}$ in order to get a binding energy of $28 \mathrm{MeV}$. The fact that in $B=1$ calculations the isoscalar nucleon radius also hits the experimental value of $0.62 \mathrm{fm}^{2}$ at constituent masses around $M_{1}=400 \mathrm{MeV}$ (see table 1 and $[5,6]$ ), leads to the conclusion, that for those constituent masses the NJL model in the hedgehog approximation is not a bad starting assumption for the evaluation of small nuclear systems. In any case, one might conclude from here that the NJL model for bigger constituent masses than $M=420 \mathrm{MeV}$ leads to a finite repulsion between two solitons at separation $d=0$. These results are in qualitative agreement with findings in the Skyrme model and in the Gell-Mann-Levy model, as far as the hedgehog symmetry is assumed. It is not clear how strongly our results depend on the choice of the hedgehog symmetry. In fact, recent investigations in the Skyrme model going beyond the hedgehog ansatz even give a negative sign for the potential energy in the case of higher winding numbers, i.e. the system with double winding is more bound than the system with single winding. It would be interesting, though extremely difficult in practice, to reconsider the system with $B=2$ in the NJL model for non-hedgehog-like configurations.

\section{Summary and conclusions}

We have investigated the baryon number equal two sector of the $S U(2) \mathrm{Nambu-}$ Jona-Lasinio model in a self-consistent way. We have raised the baryon number by 
Table 3. The potential energy $V(d=0)(\mathrm{MeV})$ for different baryon and winding numbers

\begin{tabular}{lccl}
\hline$M$ & {$\left[2 E_{B=1}-E_{B=2}\right]_{\omega=1}$} & {$\left[4 E_{B=1}-E_{B=4}\right]_{\omega=1}$} & {$\left[2 E_{B=1}-E_{B=2}\right]_{\omega=2}$} \\
\hline 370 & 136 & -274 & - \\
390 & 72 & -90 & - \\
410 & 7 & 93 & - \\
450 & 122 & 461 & - \\
560 & 523 & 1472 & 1400 \\
650 & 763 & 2300 & 1404 \\
740 & 1050 & 3130 & 1438 \\
840 & 1332 & 4046 & 1460 \\
930 & 1612 & 4877 & 1470 \\
\hline
\end{tabular}

occupying explicit positive energy quark states for different winding numbers. We have not found any solitonic solutions for fractional winding numbers. This seems to be in agreement with the quantization condition for the topological charge. In addition, we have found that self-consistent solitonic solutions do exist provided that the baryon number is larger than the winding number. Hence, in the present model the former should not always be identified with the latter. Solitonic solutions with $\omega=2$ exist only, if the constituent mass chosen is larger than some critical mass $M=580 \mathrm{MeV}$. Thus for masses around $400 \mathrm{MeV}$ required for the description of the structure of the nucleon, solitonic solutions with $\omega=2$ do not exist. Tentative numerical calculations show that each winding number has its own critical mass, which increases with increasing winding numbers. From this, we may conclude that even for higher winding and baryon numbers no solitonic solution occurs, if the constituent mass is chosen to reproduce the nucleon structure. This behaviour is quite in contrast to the topological Skyrme model, where the baryon number is identified with the winding number and solutions with higher winding numbers always exist.

In fact for moderate values of the constituent mass the system with $B>\omega$ turns out to be lower in energy than the system with $B=\omega$. In this context, the experimental deuteron binding energy and the binding energy for the $\alpha$-particle are roughly obtained at constituent masses around $M=400 \mathrm{MeV}$. Since even the isoscalar radius of the nucleon can be obtained in this range of constituent mass, the NJL model does not seem to be a bad starting approximation in order to describe nuclear properties, if $M \simeq 400 \mathrm{MeV}$ were chosen. Moreover all $B=2$ systems with constituent masses $M>420 \mathrm{MeV}$ were energetical higher than twice the $B=1$ system, may be due to the use of the hedgehog ansatz for a system, which does not need to be spherical symmetric.

It would be interesting to release the hedgehog assumption and also to consider the quantization of the rotational and isorotational degrees of freedom as a first step in trying to deduce an $\mathrm{N}-\mathrm{N}$ potential within the $\mathrm{NJL}$ model.

\section{References}

[1] Nambu Y and Jona Lasinio G 1961 Phys. Rev. 122 345; Phys. Rev. 124246

[2] Dyakonov D, Petrov V and Pobilitsa P 1988 Nucl. Phys. B 306809

[3] Meissner Th, Ruiz-Arriola E and Goeke K 1990 Z. Phys. A 33691

[4] Meissner Th, Ruiz-Arriola E, Grümmer F, Goeke K and Mavromatis H 1988 Phys. Lett. 214B 312 
Meissner Th, Grümmer F and Goeke E 1989 Phys. Lett. 227B 296

Reinhardt $\mathrm{H}$ and Wünsch $\mathrm{R} 1988$ Phys. Lett. 215B 577

[5] Meissner Th, Grümmer F, Goeke K 1990 Ann. Phys. 202,2 297

[6] Meissner Th, Goeke K 1991 Nurl. Phys. A s24 719

Goeke K, Gorski A, Grümmer F, Meissner Th, Reinhardt H and Wünsch R 1991 Phys. Lett. 256 B 321

Meissner Th and Goeke K $Z$. Phys. A at press

[7] Blotz A, Döring F, Meissner Th and Goeke K 1990 Phys.Lett. 251B 235

[8] Niemi A, Semenoff G 1986 Phys. Rep. 13599

[9] Skyrme T H R 1961 Proc. R. Soc. A 260 127; Nucl. Phys. 31556

[10] Goldstone J and Wilczek F 1981 Phys. Rev. Lett. 47986

[11] Baacke J and Schenk A 1988 Z. Phys. C 37 389; Z. Phys. C 41259

[12] Kahana S and Ripka G 1984 Nucl. Phys. A 429462

[13] Holzwarth G and Schwesinger B 1986 Rep. Progr. Phys. 49825

[14] Braaten E and Carson L 1988 Phys. Rev. D 383525

[15] Blättel B, Kunz J, Mosel U and Reitz T 1987 Nucl. Phys. A 466560

[16] Schwinger J $1351 P h y s$. Rev. 82664

[17] Dyakonov D, Petrov V and Praszalowicz M 1989 Nucl. Phys. B 32353

[18] Ripka G and Kahana S 1985 Phys. Lett. 155B 327

[19] Aitchison I and Fraser C 1985 Phys. Rev. D 312605

Aitchison I, Fraser C, Tudor E and Zuk J 1985 Phys. Lett. $165 B 162$

[20] Ebert D and Reinhard H 1986 Nucl. Phys. B 271188

[21] Jackson A, Jackson A D and Pasquier V 1984 Nucl. Phys. A 432567 Walhout T and Wambach J 1991 Preprint KFA Jülich 